\title{
MHD Natural Convective Flow Of Cu-Water Nanofluid Over A Past Infinite Vertical Plate With The Presence Of Time Dependent Boundary Condition
}

\author{
Satish Molli ${ }^{1}$, Kishan Naikoti ${ }^{2}$ \\ ${ }^{1}$ Department of Mathematics, Vignan Institute of Technology \& Sciences, Deshmukhi,Yadadri \\ Bhuvanagiri, 508284, Telangana, India \\ ${ }^{2}$ Deparment of Mathematics, Osmania University, Hyderabad, 500007, Telangana, India
}

Received: 18 June 2020; Received in revised form: 9 September 2020; Accepted: 28 September 2020; Published online 8 October 2020

(C) Published at www.ijtf.org

\begin{abstract}
In this paper, unsteady electrically conducting, incompressible, heat and mass transfer Magnetohydrodynamic free convective fluid flow with $C u$-nanoparticles over a vertical plate embedded in a porous medium and variable boundary conditions are considered. The governing PDE's have been converted to non-dimensional equations then solved by FET for velocity, temperature and concentration profiles with the influence of buoyancy force due to heat and mass transfer, Prandtl and Schmidt number, time, magnetic and chemical reaction parameter in case of pure fluid and $\mathrm{Cu}$-water nanofluid. The $\mathrm{Cu}$-water nanofluid velocity is low than pure fluid, these are presented through graphical form. Also presented the local Skin-friction coefficient, rate of heat and mass transfer and code of validation through tabular forms.
\end{abstract}

Keywords: Magnetohydrodynamic; $C u$-nanoparticles; Finite Element Technique.

\section{Introduction}

A nanofluid is a fluid having nanoparticles with various size. the fluid are colloidal suspensions of nanoparticles in the base fluids such as water, ethylene and propylene glycol. . The nanofluids have more narrative properties which make them potentially useful numerous applications in heat and mass transfer, Magnetohydrodynamic, blood flows in human body, hybrid powered engines and pharmaceutical processes etc. The enhancement of thermal conductivity and convective heat and mass transfer coefficients are compared to the base fluid. The excellent review of Nanofluid with the effect of thermal Conductivity, enhancement of Heat Transfer and comparison with experimental results were studied by Wenhua et.al[1]. Punith et. al[2] studied mixed convective flow of an incompressible nanofluid along an inclined vertical plate embedded in a porous medium through finite element method and they concentrated on development of steady state boundary layer fluid 
Satish and Kishan

International Journal of Thermofluid Science and Technology (2020), Volume 7, Issue 4, Paper No. 070404

flow with effect of heat transfer in nanofluid. Khan and Aziz [3] analysed the boundary stratum of a nanofluid flow over a past vertical surface with the presence of a constant heat flux. Putra et.al [4] has been studied natural convective flow of nanofluids $\mathrm{Al}_{2} \mathrm{O}_{3}$-water and water-based copper oxide with effect of heat transfer. Kuznetsov and Nield [5] studied free convective border line stratum flow of a nanofluid over the vertical plate with the influence of thermophoresis and Brownian motion. Gorla and Chamkha [6] presented the free convective boundary stratum of nanofluid flow with the presence of porous medium. Krishnamurthy et.al[7] have investigated magnetohydrodynamic nanofluid flow over an exponential stretched sheet with the influence of viscous dissipation. The authors applied Runge-kutta-Fehlberg method to solve the non-dimensionless governing equations using the MAPLE software with the influence of various parameters. Khan and Pop [8] studied the boundary layer flow of a nanofluid past a stretching sheet by considering the Brownian diffusion and thermophoresis effects. Mackolil and mahenthesh [9] are studied the exact and statistical analysis of nanofluid and Casson fluid flow over a past an infinite vertical plate with the presence of mass and heat flux. Raza et al. [10] studied the hydromagnetic nanofluid in three-dimensional flow with a rotating channel and the authors studied simultaneously various effects on nanoparticles . Aziz and Khan [11] analyzed the behavior of of a nanofluid with convective flow over a heated vertical plate. Ganga et al. [12] found velocity and energy of nanofluid over a vertical plate with the effect of internal heat generation/absorption. Ali et al. [13] studied an Magnetohydrodynamic nanofluid flow over a permeable vertical plate with heat and mass transfer. Kandelousi [14] also studied the nanofluid flow through permeable channel with heat transfer effect. Tiwari and Das [15] presented the numerical study of nanofluid flow which is filled in a two-sided lid-driven differentially heated square cavity. Sheikholeslami et.al[16] studied $\mathrm{Cu}$-water nanofluid on a stretched sheet through the HAM method. M K Nayak et.al [17] investigated a non-uniform heat source/sink on mixed convective flow of nanofluid flow on inclined stretched plate which is in ring form. The governing non dimensional equations are solved by RK method and shooting technique for the analysis of fluid velocity, temperature and rate of heat transfer from the plate. A.K. Patra et.al [18] reported a review on viscosity of nanofluids. S. Mishra et.al [19] done a comprehensive review on thermal conductivity of fluid. Nayak [20] studied a homogenous and heterogeneous reactions on three-dimensional flow of $\mathrm{Cu}$-water nanofluid over an exponential stretching sheet with the presence of variable magnetic field and thermal radiation. He attempted RK method and shooting technique to solve the dimensionless equations for the behavior of the nanofluid. Kalidas et. al [21] investigated the behavior of $\mathrm{Cu}$-water nanofluid flow on a vertical plate with the influence of few parameters. but the authors not explored with time dependent boundary conditions. Pattnaik and Biswal [22] have been studied MHD free convective fluid flow on a vertical plate with the effect of heat source, chemical reaction, time dependent concentration and temperature. The author's analyzed the fluid behavior through analytical method, but not explored behavior of nanofluid.

In view of the above investigation, we have been studied the unsteady Magnetohydrodynamic Natural convective heat and mass transfer of pure and $\mathrm{Cu}$-water nanofluid over a vertical plate embedded in a porous media with the effect of emerging parameters with the presence of chemical reaction, time dependent concentration and temperature. The governing equations are solved by FEM, have been maintaining an 
Satish and Kishan

International Journal of Thermofluid Science and Technology (2020), Volume 7, Issue 4, Paper No. 070404

accuracy $10^{-5}$. Presented the comparison and behavior of pure fluid and $\mathrm{Cu}$-water nanofluid through the graphical form.

\section{Formulation of the problem}

The unsteady Magnetohydrodynamic, incompressible, electrically conducting, viscous, boundary layer flow of nanofluid over a vertical plate embedded in a porous media in presence of chemical reaction, For this investigation, we made the following assumptions:

i. The fluid flow in unidirectional and restricted to the $y^{\prime}>0$

ii. The $x^{\prime}$-axis is taken along the plate and $y^{\prime}$-axis is normal to the plate.

iii. The temperature of surface of the plate is raised to uniform temperature $T_{w}^{\prime}$ at $t^{\prime}>0$ and species concentration of surface of the plate is raised to uniform species concentration $C_{w}^{\prime}$ and is maintained thereafter.

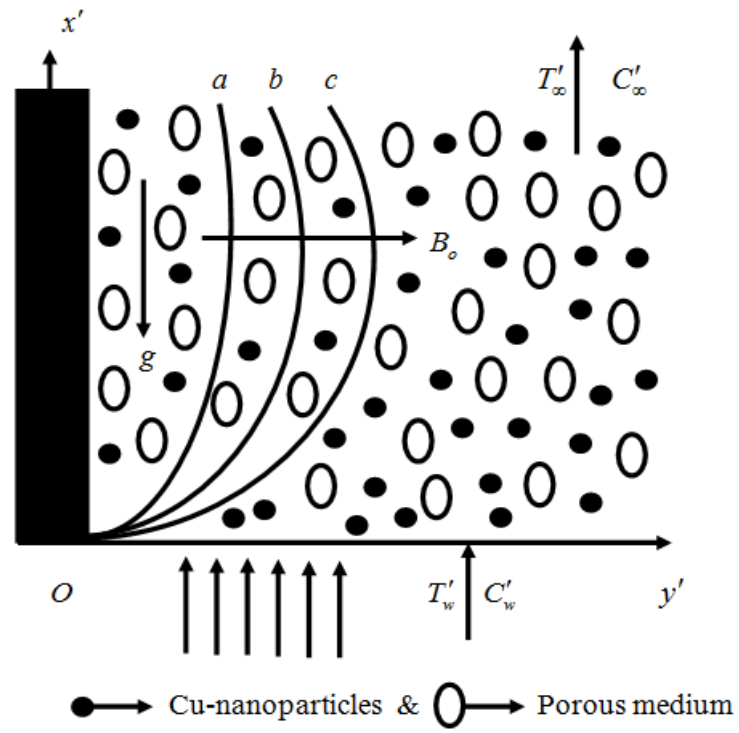

$a$--- Momentum boundary layer, $b$--- Thermal boundary layer, $c$--- Concentration boundary layer

Fig. 1. Geometry of the problem

iv. The first order chemical reaction porous medium are considered.

v. the fluid flow through the porous media satisfies the Darcy's law, which accounts for the drag exerted by the porous medium.

vi. Magnetic field of intensity $B_{0}$ is applied on the plate and it should be normal to the plate.

vii. During $t^{\prime} \leq 0$, both the fluid and stationary plate are kept at a uniform temperature $T_{\infty}^{\prime}$, thespecies concentration of the surface of the plate and at each point in the fluid are kept at a uniform concentration $C_{\infty}^{\prime}$.

viii. The magnetic Reynolds number is assumed to be very small such that the thickness of the magnetic boundary-layer is large, compared to the applied magnetic field, so that the induced magnetic field effect is negligible.

ix. Both the fluid phase and nanoparticles are in thermal equilibrium state.

x. There is no applied voltage which implies the absence of an electric field. 
xi. Energy dissipation such as viscous dissipation and Joulian dissipation are also neglected as we are considering free convective and slow flow neglecting inertia terms in the transport equations.

xii. The Hall effect is neglected.

xiii. Thermal diffusion and Diffusion thermo effects are neglected due to the low thermal and concentration gradient.

From the above assumptions, we obtained the conservation of momentum, energy and concentration equations for an unsteady flow of $\mathrm{Cu}$-nanofluid in $t^{\prime}$ and $y^{\prime}$.

Momentum Equation:

$\rho_{n f}\left(\frac{\partial u^{\prime}}{\partial t^{\prime}}\right)=\mu_{n f}\left(\frac{\partial^{2} u^{\prime}}{\partial y^{\prime 2}}\right)-\sigma_{n f} B_{0}{ }^{2} u^{\prime}+(\rho \beta)_{n f} g\left(T^{\prime}-T_{\infty}^{\prime}\right)+\left(\rho \beta^{*}\right)_{n f} g\left(T^{\prime}-T_{\infty}^{\prime}\right)-\frac{\mu_{n f} u^{\prime}}{K^{\prime}}$

Energy Equation:

$\frac{\partial T^{\prime}}{\partial t^{\prime}}=\frac{\kappa_{n f}}{\left(\rho C_{p}\right)_{n f}}\left(\frac{\partial^{2} T^{\prime}}{\partial y^{\prime 2}}\right)-\frac{Q_{o}}{\left(\rho C_{p}\right)_{n f}}\left(T^{\prime}-T_{\infty}^{\prime}\right)$

Species Diffusion Equation:

$\frac{\partial C^{\prime}}{\partial t^{\prime}}=D_{B} \frac{\partial^{2} C^{\prime}}{\partial y^{\prime 2}}-K_{r}^{\prime}\left(C^{\prime}-C_{\infty}^{\prime}\right)$

In nanofluids, the expressions of density $\left(\rho_{n f}\right)$, thermal expansion coefficient $(\rho \beta)_{n f}$ and heat capacitance $\left(\rho C_{p}\right)_{n f}$ as

$$
\left.\begin{array}{l}
\rho_{n f}=(1-\phi) \rho_{f}+\phi \rho_{s},(\rho \beta)_{n f}=(1-\phi)(\rho \beta)_{f}+\phi(\rho \beta)_{s}, \\
\left(\rho \beta^{*}\right)_{n f}=(1-\phi)\left(\rho \beta^{*}\right)_{f}+\phi\left(\rho \beta^{*}\right)_{s},\left(\rho C_{p}\right)_{n f}=(1-\phi)\left(\rho C_{p}\right)_{f}+\phi\left(\rho C_{p}\right)_{s}
\end{array}\right\}
$$

The thermal and electrical conductivity of the nanofluid(Hamilton and Crosser [23]) model as

$$
\begin{aligned}
& \kappa_{n f}=\kappa_{f}\left[\frac{\kappa_{s}+2 \kappa_{f}-2 \phi\left(\kappa_{f}-\kappa_{s}\right)}{\kappa_{s}+2 \kappa_{f}+2 \phi\left(\kappa_{f}-\kappa_{s}\right)}\right], \mu_{n f}=\frac{\mu_{f}}{(1-\phi)^{2.5}} \\
& \sigma_{n f}=\sigma_{f}\left[1+\frac{3(\sigma-1) \phi}{\sigma+2-(\sigma-1) \phi}\right], \sigma=\frac{\sigma_{s}}{\sigma_{f}}
\end{aligned}
$$

Subject to the boundary conditions are

$$
\begin{aligned}
& t^{\prime} \leq 0:\left\{u^{\prime}\left(y^{\prime}, t^{\prime}\right)=0, T^{\prime}\left(y^{\prime}, t^{\prime}\right)=T_{\infty}^{\prime}, C^{\prime}\left(y^{\prime}, t^{\prime}\right)=C_{\infty}^{\prime} \text { for all } y^{\prime}\right. \\
& t^{\prime}>0:\left\{\begin{array}{l}
u^{\prime}\left(y^{\prime}, t^{\prime}\right)=U_{o}\left(a^{\prime} t^{\prime}\right), T^{\prime}\left(y^{\prime}, t^{\prime}\right)=T_{\infty}^{\prime}+\frac{\left(T_{w}^{\prime}-T_{\infty}^{\prime}\right) U_{o}^{2} t^{\prime}}{v}, C^{\prime}\left(y^{\prime}, t^{\prime}\right)=C_{\infty}^{\prime}+\left(C_{w}^{\prime}-C_{\infty}^{\prime}\right) \exp \left(\frac{U_{o}^{2} t^{\prime}}{v}\right) \text { at } y^{\prime}=0 \\
u^{\prime}\left(y^{\prime}, t^{\prime}\right)=0, T^{\prime}\left(y^{\prime}, t^{\prime}\right) \rightarrow T_{\infty}^{\prime}, C^{\prime}\left(y^{\prime}, t^{\prime}\right) \rightarrow C_{\infty}^{\prime} \text { as } y^{\prime} \rightarrow \infty
\end{array}\right\}
\end{aligned}
$$

On introducing the following non-dimensional quantities 


$$
\begin{aligned}
& u=\frac{u^{\prime}}{U_{o}}, y=\frac{y^{\prime} U_{o}}{v_{f}}, t=\frac{t^{\prime} U_{o}^{2}}{v_{f}}, \theta=\frac{T^{\prime}-T_{\infty}^{\prime}}{T_{w}^{\prime}-T_{\infty}^{\prime}}, C=\frac{C^{\prime}-C_{\infty}^{\prime}}{C_{w}^{\prime}-C_{\infty}^{\prime}}, K=\frac{K^{\prime} U_{o}^{2}}{v_{f}^{2}}, M=\frac{\sigma_{f} v_{f} B_{o}^{2}}{\rho_{f} U_{o}^{2}}, S=\frac{Q_{o} v_{f}}{\left(\rho C_{p}\right)_{f} U_{o}^{2}}, \\
& a=\frac{v_{f} a^{\prime}}{U_{0}^{2}}, \operatorname{Pr}=\frac{\left(\rho C_{p}\right)_{f} v_{f}}{\kappa_{f}}, G r=\frac{v_{f} g \beta_{f}\left(T_{w}^{\prime}-T_{\infty}^{\prime}\right)}{U_{o}^{3}}, S c=\frac{v_{f}}{D_{B}}, K r=\frac{K_{r}^{\prime} v_{f}}{U_{o}^{2}}, G c=\frac{v_{f} g \beta_{f}^{*}\left(C_{w}^{\prime}-C_{\infty}^{\prime}\right)}{U_{o}^{3}}
\end{aligned}
$$

Then Eqs. (1), (2) and (3) transform to the following non-dimensional forms

$$
\begin{aligned}
& \frac{\partial u}{\partial t}=A_{1} \frac{\partial^{2} u}{\partial y^{2}}+A_{2} G r \theta+A_{3} G c C-A_{4}\left(M+\frac{1}{K}\right) u \\
& \frac{\partial \theta}{\partial t}=\frac{1}{\operatorname{Pr}} \frac{\partial^{2} \theta}{\partial y^{2}}-A_{5} \theta \\
& \frac{\partial C}{\partial t}=\frac{1}{S c} \frac{\partial^{2} C}{\partial y^{2}}-K r C
\end{aligned}
$$

Where $x_{1}=(1-\phi)+\phi\left(\frac{\rho_{s}}{\rho_{f}}\right), x_{2}=(1-\phi)+\phi\left(\frac{(\rho \beta)_{s}}{(\rho \beta)_{f}}\right), x_{3}=(1-\phi)+\phi\left(\frac{\left(\rho \beta^{*}\right)_{s}}{\left(\rho \beta^{*}\right)_{f}}\right)$,

$$
\begin{aligned}
& x_{4}=\left[1+\frac{3(\sigma-1) \phi}{\sigma+2-(\sigma-1) \phi}\right], x_{5}=(1-\phi)+\phi\left(\frac{\left(\rho C_{p}\right)_{s}}{\left(\rho C_{p}\right)_{f}}\right), x_{6}=\frac{\kappa_{n f}}{\kappa_{f}}=\left[\frac{(1+2 \phi)+(2-2 \phi)\left(\frac{\kappa_{f}}{\kappa_{s}}\right)}{(1-2 \phi)+(2+2 \phi)\left(\frac{\kappa_{f}}{\kappa_{s}}\right)}\right], \\
& A_{1}=\frac{1}{(1-\phi)^{2.5} x_{1}}, A_{2}=\frac{x_{2}}{x_{1}}, A_{3}=\frac{x_{3}}{x_{1}}, A_{4}=\frac{x_{4}}{x_{1}}, A_{5}=\frac{S}{x_{5}}
\end{aligned}
$$

The correspond ing boundary conditions (7) in non-dimensional forms are

$$
\begin{array}{ll}
t \leq 0: & u=0, \theta=0, C=0 \text { for all } y \\
t>0: & \left\{\begin{array}{l}
u=a t, \theta=t, C=\exp (t) \text { at } y=0 \\
u \rightarrow 0, \theta \rightarrow 0, C \rightarrow 0 \text { as } y \rightarrow \infty
\end{array}\right\}
\end{array}
$$

All the physical variables are defined in the nomenclature. The skin-friction at the plate, which is the non-dimensional form is given by

$$
C f=\frac{\left(\frac{\partial u^{\prime}}{\partial y^{\prime}}\right)_{y^{\prime}=0}}{\rho_{f} U_{o} v_{f}}=\frac{1}{(1-\phi)^{2.5}}\left(\frac{\partial u}{\partial y}\right)_{y=0}
$$

The rate of heat transfer coefficient, which is the non-dimensional form in terms of the Nusselt number $(\mathrm{Nu})$ is given by

$$
N u=-\left(\frac{x^{\prime}}{T_{w}^{\prime}-T_{\infty}^{\prime}}\right)\left(\frac{\partial T^{\prime}}{\partial y^{\prime}}\right)_{y^{\prime}=0} \Rightarrow N u \operatorname{Re}_{x}^{-1}=-\left(\frac{\kappa_{n f}}{\kappa_{f}}\right)\left(\frac{\partial \theta}{\partial y}\right)_{y=0}
$$

The rate of mass transfer coefficient, which is the non-dimensional form in terms of the Sherwood number $(S h)$, is given by 


$$
S h=-\left(\frac{x^{\prime}}{C_{w}^{\prime}-C_{\infty}^{\prime}}\right)\left(\frac{\partial C^{\prime}}{\partial y^{\prime}}\right)_{y^{\prime}=0} \Rightarrow S h \operatorname{Re}_{x}^{-1}=-\left(\frac{\partial C}{\partial y}\right)_{y=0}
$$

Where $\operatorname{Re}=\frac{U_{o} x^{\prime}}{v_{f}}$ is the local Reynolds number.

\section{Numerical Solutions By Finite Element Method}

The finite element procedure (FEM) is a numerical and computer based method ([24] and [25]) of solving a collection of practical engineering problems that happen in different fields such as, in heat transfer, fluid mechanics and many other fields. It is recognized by developers and consumers as one of the most influential numerical analysis tools ever devised to analyze complex problems of engineering. The superiority of the method, its accuracy, simplicity, and computability all make it a widely used apparatus in the engineering modelling and design process. It has been applied to a number of substantial mathematical models, whose differential equations are solved by converting them into a matrix equation. The primary feature of FEM is its ability to describe the geometry or the media of the problem being analyzed with huge flexibility. This is because the discretization of the region of the problem is performed using highly flexible uniform or non uniform pieces or elements that can easily describe complex shapes. The method essentially consists in assuming the piecewise continuous function for the results and getting the parameters of the functions in a manner that reduces the fault in the solution. The steps occupied in the finite element analysis areas follows.

Step-1: Discretization of the Domain: The fundamental concept of the FEM is to divide the region of the problem into small connected pieces, called finite elements. The group of elements is called the finite element mesh. These finite elements are associated in a non overlapping manner, such that they completely cover the entire space of the problem.

Step-2: Invention of the Element Equations:

I). A representative element is secluded from the mesh and the variational formulation of the given problem is created over the typical element.

II). Over an element, an approximate solution of the variational problem is invented, and by surrogating this in the system, the element equations are generated.

III). The element matrix, which is also known as stiffness matrix, is erected by using the element interpolation functions.

Step-3: Assembly of the Element Equations: The algebraic equations so achieved are assembled by imposing the inter element continuity conditions. This yields a large number of mathematical equations known as the global finite element model, which governs the whole domain.

Step-4: Imposition of the Boundary Conditions: On the accumulated equations, the Dirichlet's and Neumann boundary conditions (12) are imposed.

Step-5: Solution of Assembled Equations: The assembled equations so obtained can be solved by Gauss elimination technique and the final matrix equation can be solved by iterative technique. For computational purposes, the coordinate $y$ varies from 0 to 10 , where $y_{\max }$ represents infinity external to the momentum, energy and concentration edge layers.

In one-dimensional space, linear elements are taken. The entire flow province is divided into 11000 quadratic elements of equal size. Each element is two-noded, and therefore the whole domain contains 21001 nodes. At each node, three functions are to be 
Satish and Kishan

International Journal of Thermofluid Science and Technology (2020), Volume 7, Issue 4, Paper No. 070404

evaluated; hence, after assembly of the element equations, we acquire a system of 63003 equations which are non-linear. Therefore, an iterative scheme must be developed in the solution. After striking the boundary conditions, a system of equations has been obtained which is solved mathematically by the Gauss elimination method while maintaining a correctness of 0.00001 . A convergence criterion based on the relative difference between the present and preceding iterations is employed. When these differences satisfy the desired correctness, the solution is assumed to have been congregated and iterative process is terminated. The Gaussian quadrature is applied for solving the integrations. The computer cryptogram of the algorithm has been performed in MATLAB running on a PC. Excellent convergence was completed for all the results.

\section{Results and Discussion}

The non dimensional system of Partial differential equations (9) -(10) for momentum, energy and concentration based on the properties of nanofluids (Table-1) with suitable boundary conditions (12) is solved with the effect of pertinent parameters such as the Grashof number $(G r)$ and $(G c)$ for heat transfer and mass transfer, Permeability $(K)$, Magnetic field $(M)$, Heat source $(S)$ and Chemical reaction $(K r)$ parameters, Prandtl $(\operatorname{Pr})$ and Schmidt $(S c)$ number. The flow behaviour and the results are illustrated through graphical form from Fig.2 to Fig. 16. Also presented the local skin-friction coefficient and local Nusselt and Sherwood numbers through tabulated form.

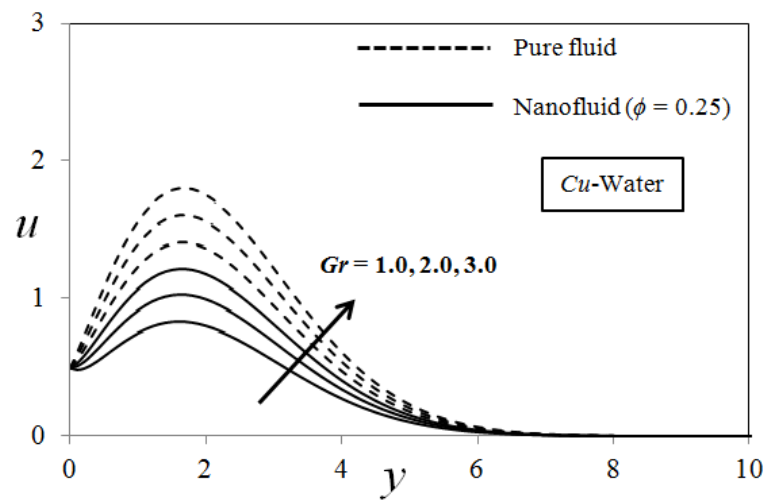

Fig. 2. $G r$ effect on velocity profiles

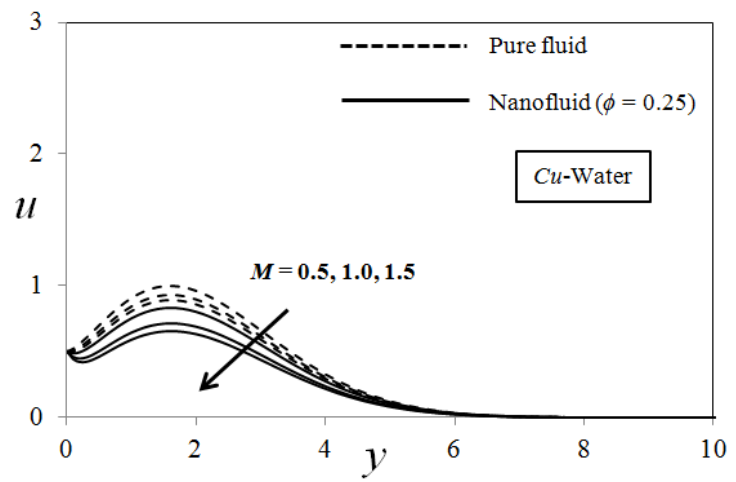

Fig. 4. $M$ effect on velocity profiles

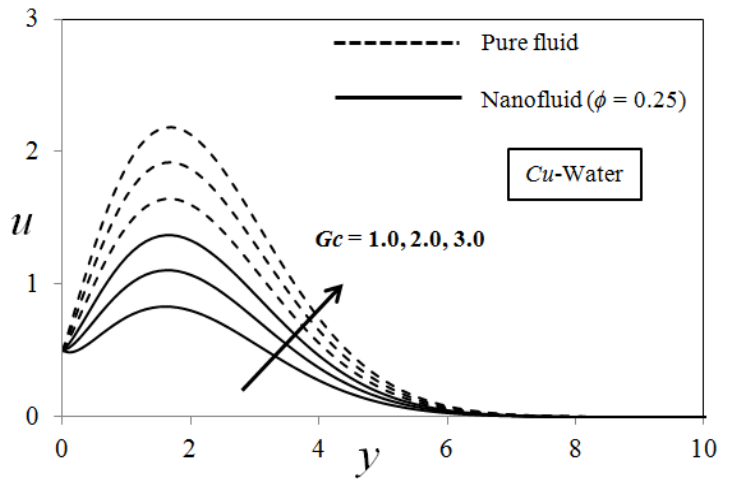

Fig. 3. $G c$ effect on velocity profiles

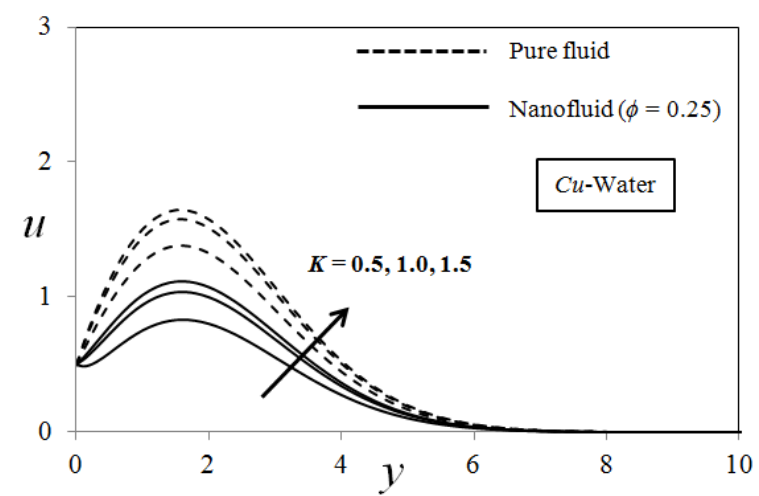

Fig. 5. $K$ effect on velocity profiles

Fig.2 and Fig.3 shows the influence of $G r$ and $G c$ on pure and nanofluid velocity profile. The pure fluid and $\mathrm{Cu}$-water nanofluid velocity raises as increasing of Grashof 
Satish and Kishan

International Journal of Thermofluid Science and Technology (2020), Volume 7, Issue 4, Paper No. 070404

numbers $G r$ and $G c$ for heat and mass transfer. Physically, the relationship between buoyancy and viscous force is called the Grashof number, the asymmetry in the floating forces controls the momentum boundary layer, because of that, an expansion in the boundary layer thickness is high. Also, we observed that in case of $\mathrm{Cu}$-nanoparticles thermal boundary layer is very thicker than the regular fluid. Fig. 4 signify the velocity of $\mathrm{Cu}$-water nanofluid and pure fluid with the influence of Magnetic parameter M. As increasing of Magnetic parameter, velocity of $\mathrm{Cu}$-water nanofluid decreases due to produces an opposing force (Lorentz force), which reduces the motion of the fluid and creates a marked resistance.

The velocity of $\mathrm{Cu}$-water nanofluid and pure fluid decreases as escalating of the permeability parameter $K$. The presence of porous media increases the resistance to flow, resulting in a decrease in the thickness of the thermal boundary layer. It is shown in the Fig.5.The effect of the porous medium on the boundary layer reduction is considerable. The influence of Prandtl number $\mathrm{Pr}$ on velocity and temperature of $\mathrm{Cu}$-water nanofluid and pure fluid is shown in the Fig. 6 and 7 respectively. $\mathrm{Cu}$-water nanofluid and pure fluid velocity and temperature decreasing as increasing of Prandtl number Pr. Prandtl number Pr is the relationship between the viscosity and thermal conductivity. The high Prandtl number represents the high viscosity and low thermal conductivity. we know that at high viscosity, the velocity as well as temperature of fluid flow is low. because of that, the $\mathrm{Cu}$-water nanofluid and pure fluid velocity and temperature decreasing as increasing of Prandtl number Pr.The effect of heat source parameter on velocity as well as temperature of $\mathrm{Cu}$-water nanofluid and pure fluid presented in Figs. 8 and 9 respectively. Those graphs shows that, enhancing of heat source parameter $S$, both $\mathrm{Cu}$-water nanofluid and pure fluid velocity and temperature profiles are decreasing at the boundary and thermal boundary layers of nanofluid respectively.

Table-1.: Thermo Physical Properties

\begin{tabular}{ccc}
\hline Physical Properties & Water (base fluid) & $C u$ (Copper) \\
\hline$C_{p}(J / K g-K)$ & 4179 & 385 \\
$\rho\left(K g / m^{3}\right)$ & 997.1 & 893.3 \\
$\kappa(W / m-K)$ & 0.613 & 400 \\
$\rho \times 10^{-5}(1 / K)$ & 21 & 1.67 \\
\hline
\end{tabular}

Fig.10 and Fig.11 shows the $\mathrm{Cu}$-water nanofluid and pure fluid velocity and concentration with the effect of Schmidt number $S c$. Taken the few values for Sc such as, $S c$ $=0.22$ (Hydrogen), $S c=0.60$ (Water vapour), $S c=0.78$ (Ammonia). Schmidt number $S c$ is the relative amount of viscous diffusion rate to mass diffusion rate. As increasing of Schmidt number viscous diffusion rate high than mass diffusion rate. At the high viscous diffusion rate, velocity of fluid is low and at low mass diffusion rate, concentration of fluid is low. Hence the velocity and concentration of $\mathrm{Cu}$-water nanofluid and pure fluid are falling as growing of Schmidt number. The effect of chemical reaction parameter on velocity and concentration of $\mathrm{Cu}$-water nanofluid and pure fluid illustrated in Fig. 12 and 13. As enhancing of the chemical reaction parameter leads to the reduction in the concentration and motion of the fluid is low. Therefore, an increasing of chemical reaction parameter, the fluid velocity and concentration are decreased . 
Table-2. Variation and Comparison of Skin-friction with the variation of parameters.

\begin{tabular}{cccccccccc}
\hline & & & & & & & $\tau^{*}$ Pattnaik & \multicolumn{2}{c}{$\boldsymbol{C f}$} \\
\cline { 7 - 9 } & $\boldsymbol{G} \boldsymbol{c}$ & $\boldsymbol{M}$ & $\boldsymbol{K}$ & $\mathbf{P r}$ & $\boldsymbol{S}$ & $\boldsymbol{K} \boldsymbol{r}$ & $\begin{array}{c}\text { and Biswal } \\
{[22]}\end{array}$ & Pure fluid & Cu-water \\
\hline 0.5 & 0.1 & 2.0 & 100.0 & 1.6 & 0.8 & 0 & 1.063313 & 1.063313 & 1.217840 \\
1.0 & 0.1 & 2.0 & 100.0 & 1.6 & 0.8 & 0 & 1.473819 & 1.473819 & 1.687521 \\
0.5 & 0.5 & 2.0 & 100.0 & 1.6 & 0.8 & 0 & 1.994610 & 1.994610 & 2.017628 \\
0.5 & 0.1 & 3.0 & 100.0 & 1.6 & 0.8 & 0 & 1.364556 & 1.364556 & 1.587496 \\
0.5 & 0.1 & 2.0 & 0.50 & 1.6 & 0.8 & 0 & 0.230490 & 0.230490 & 0.387641 \\
0.5 & 0.1 & 2.0 & 100.0 & 2.0 & 0.8 & 0 & 2.591956 & 2.591956 & 2.795612 \\
0.5 & 0.1 & 2.0 & 100.0 & 1.6 & 0.5 & 0 & 2.360807 & 2.360807 & 2.495217 \\
0.5 & 0.1 & 2.0 & 100.0 & 1.6 & 0.8 & 1 & - & 0.918352 & 1.074925 \\
\hline
\end{tabular}

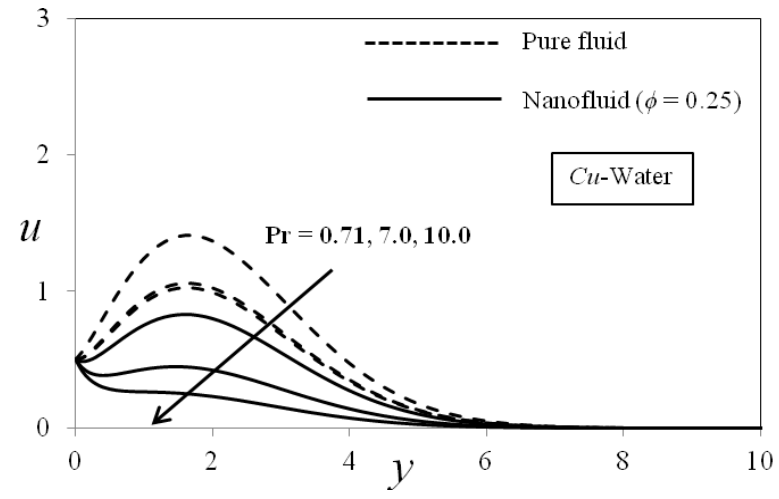

Fig. 6. Pr effect on velocity profiles

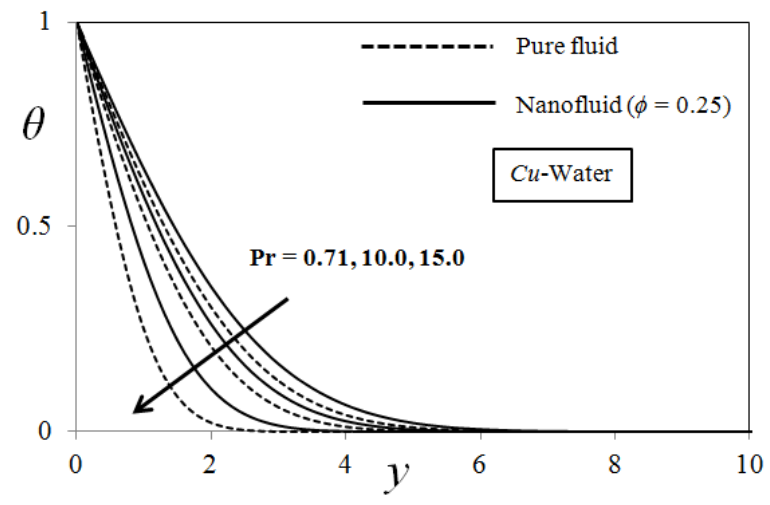

Fig. 7. Pr effect on temperature profiles

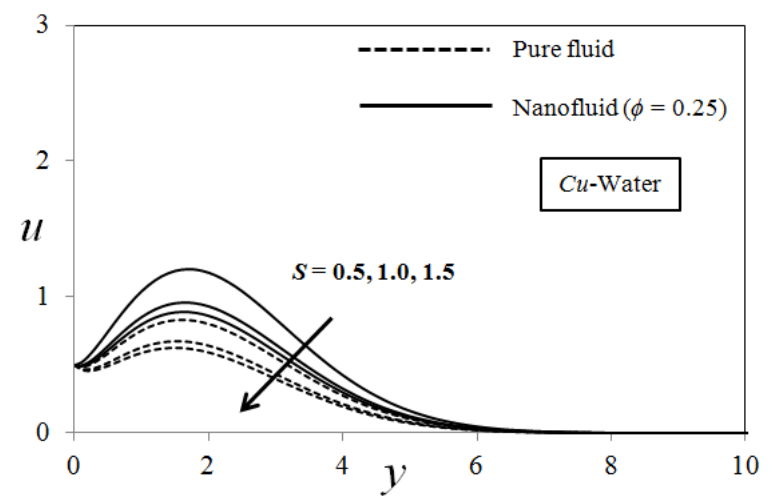

Fig. 8. $S$ effect on velocity profiles

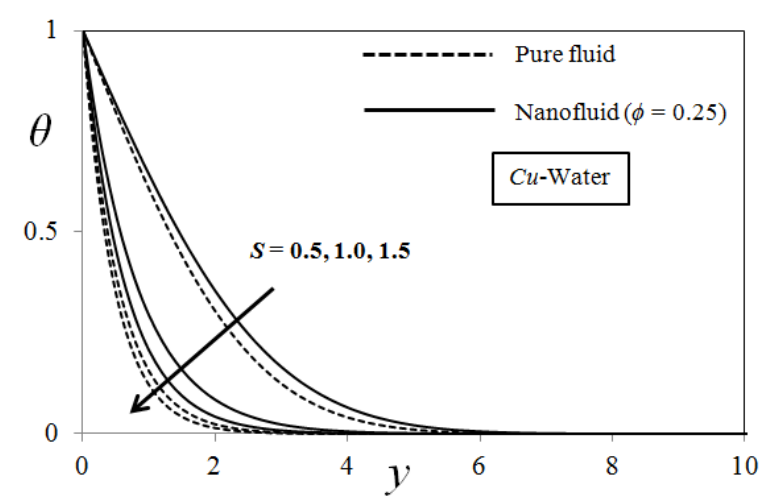

Fig. 9. $S$ effect on temperature profiles 
Table 3: Variation and comparison of Nusselt number

\begin{tabular}{ccccc}
\hline & & \multirow{2}{*}{ Nu (Pattnaik } & \multicolumn{2}{c}{ Nu } \\
\cline { 4 - 5 } & $S$ & and Bis wal [22]) & Pure fluid & Cu-water \\
\hline 1.6 & 0.8 & 0.61349617 & 0.61349617 & 0.74895223 \\
1.8 & 0.8 & 1.75705764 & 1.75705764 & 1.84521082 \\
1.6 & 0.5 & 1.75021931 & 1.75021931 & 1.95153764 \\
\hline
\end{tabular}

Table 4: Variation of Sherwood number

\begin{tabular}{cccc}
\hline $\boldsymbol{S c}$ & $\boldsymbol{K} \boldsymbol{r}$ & $\boldsymbol{t}$ & $\boldsymbol{S h}$ \\
\hline 0.22 & 1.00 & 1.00 & 2.387464 \\
$\mathbf{0 . 6 0}$ & 1.00 & 1.00 & 1.284939 \\
0.22 & $\mathbf{2 . 0 0}$ & 1.00 & 1.128758 \\
0.22 & 1.00 & $\mathbf{2 . 0 0}$ & 2.974618 \\
\hline
\end{tabular}

An impact of time on velocity, temperature and concentration of $\mathrm{Cu}$-water nanofluid and pure fluid are illustrated in the figures ( Figs. 14-16). From those figures, Cu-water nanofluid and pure fluid velocity, temperature and concentration are raised as an enhancement of time. The effects of Grashof number for heat transfer $G r$, Grashof number for mass transfer $G c$, Magnetic field parameter $M$, Permeability parameter $K$, Schmidt number $S c$, Prandtl number Pr, Heat source parameter $S$, Chemical reaction parameter $K r$ and Time $t$ on skin-friction coefficient is presented in table 5 with the help of numerical values. From this table, it is observed that, the numerical values of skin-friction coefficient is increasing under the increasing of Grashof number for heat tra nsfer Gr, Grashof number for mass transfer $G c$, Permeability parameter $K$, Time $t$ while it decreasing under the increasing of Magnetic field parameter $M$, Schmidt number $S c$, Prandtl number Pr, Heat source parameter $S$ and Chemical reaction parameter $K r$.

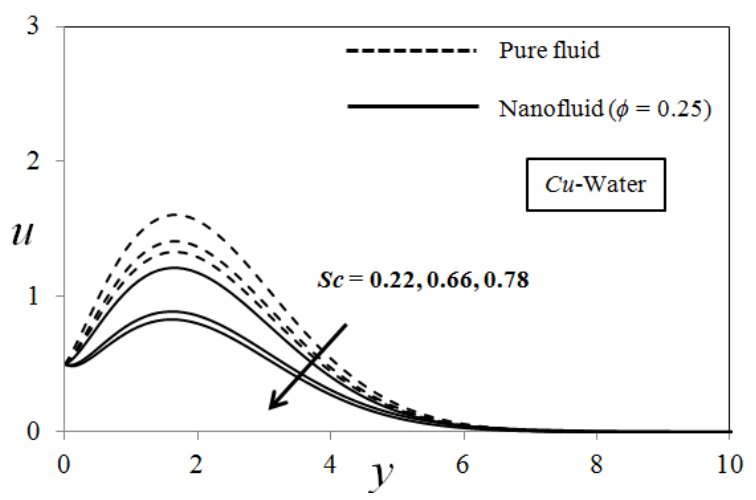

Fig. 10. $S c$ effect on velocity profiles

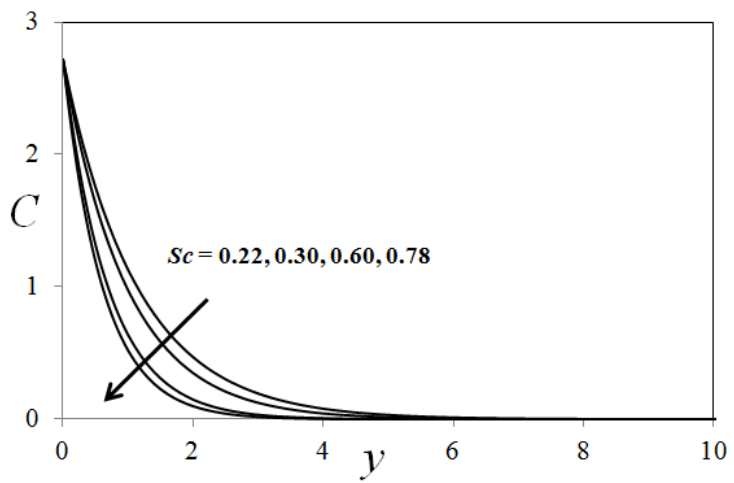

Fig. 11. $S c$ effect on concentration profiles 


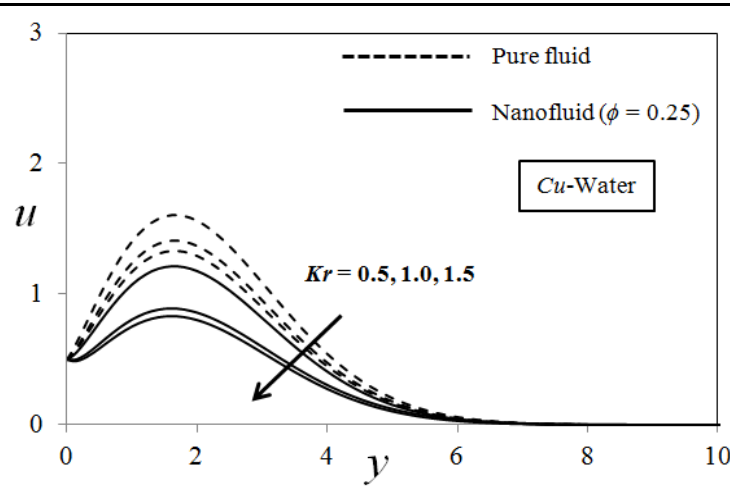

Fig. 12. $K r$ effect on velocity profiles

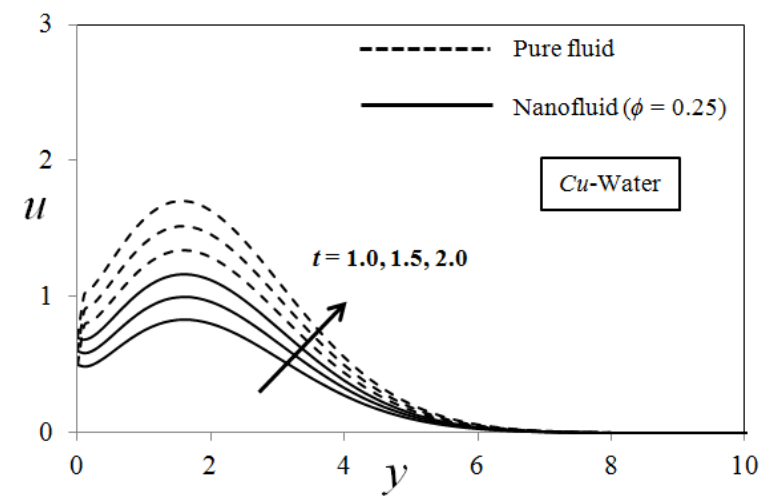

Fig. 14. $t$ effect on velocity profiles

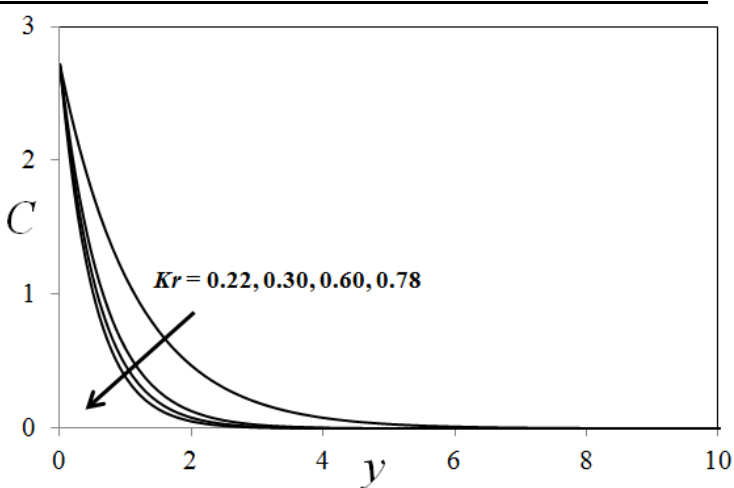

Fig. 13. $K r$ effect on concentration profiles

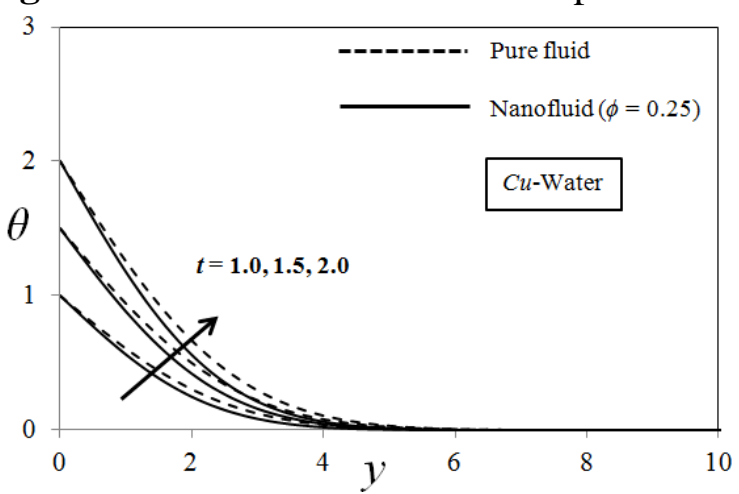

Fig. 15. $t$ effect on temperature profiles

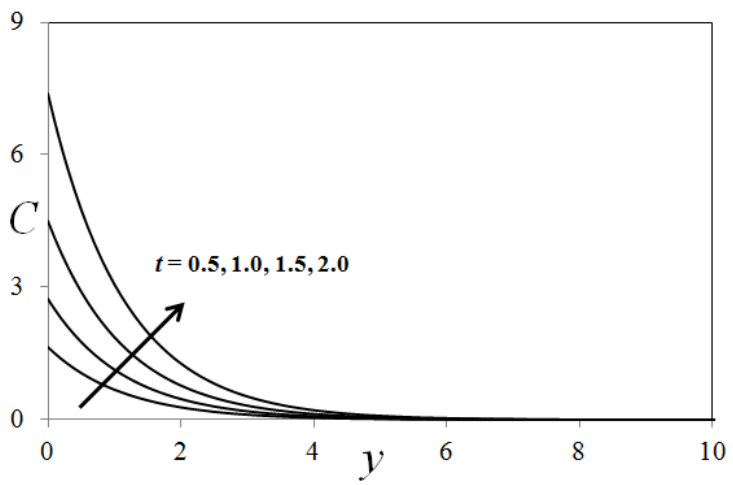

Fig. 16. $t$ effect on concentration profile

Table 2-4 shows the Skin-friction, rate of heat and mass transfer of $\mathrm{Cu}$-water nanofluid and pure fluid and also presented the comparison of present results with existed closed form solutions of Pattnaik and Biswal [22] for existing and correctness of Finite element method. The results are good agreement to the results of Pattnaik and Biswal [22]. The effects of Grashof number for heat transfer $G r$, Grashof number for mass transfer $G c$, Magnetic field parameter $M$, Permeability parameter $K$, Schmidt number $S c$, Prandtl number Pr, Heat source parameter $S$, Chemical reaction parameter $K r$ and Time $t$ on skin-friction coefficient is presented in Table 2 with the help of numerical values. From this table, it is observed that, the numerical values of skin-friction coefficient is increasing under the increasing of Grashof number for heat transfer $G r$, Grashof number for mass trans fer $G c$, Permeability parameter $K$, 
Satish and Kishan

International Journal of Thermofluid Science and Technology (2020), Volume 7, Issue 4, Paper No. 070404

Time $t$ Magnetic field parameter $M$, Schmidt number $S c$ and Prandtl number Pr while it decreasing under the increasing of Heat source parameter $S$ and Chemical reaction parameter $K r$.

The effects Prandtl number Pr and Heat source parameter $S$ on rate of heat transfer coefficient in terms of Nusselt number is discussed in Table 3. From this table, it is observed that, the numerical values of Nusselt number coefficient is increasing with the increasing of Prandtl number Pr while decreasing as increasing of Heat source parameter $S$. Skin-friction and Nusselt number values of $\mathrm{Cu}$-water nanofluid are high than the pure fluid. Table 4 , shows the numerical values of rate of mass transfer coefficient in terms of Sherwood number coefficient for different values of Schmidt number $S c$, Chemical reaction parameter $K r$ and Time $t$. From this table, it is observed that Sherwood number coefficient is increasing with increasing values of Time $t$ and decreasing with increasing of Schmidt number $S c$ and Chemical reaction parameter $K r$.

\section{Conclusion}

A numerical Finite element technique has been employed to study of unsteady-state unidirectional flow of pure and $\mathrm{Cu}$-water nanofluid. The effects of various emerging governing parameters on the velocity, temperature and concentration of fluid flow characteristics are drawn.

i. The velocity of $\mathrm{Cu}$-water nanofluid is low than pure fluid while reversible behaviour observed in temperature profile.

ii. The velocity, concentration and temperature fields are increased by increasing of the time

iii. The fluid velocity increases as increasing of Grashof number, Permeability parameter while decreasing as increasing of Prandtl number, Magnetic parameter, Schmidt number and $\mathrm{Chemical}$ reaction parameters.

iv. The fluid concentration decreases with increasing of Schmidt number and Chemical reaction parameters.

v. The fluid temperature decreases with an increase Prandtl number and heat source parameter.

vi. Skin-friction and Nusselt number values of $\mathrm{Cu}$-water nanofluid are high than the pure fluid.

vii. On code validation of this work, the present results are well agreed with the closed form results of Pattnaik and Biswal [22].

\section{Nomenclature:}

\begin{tabular}{|c|c|c|}
\hline$T_{\infty}^{\prime}$ & $\begin{array}{l}\text { Fluid temperature far away from the plate } \\
(\mathrm{K})\end{array}$ & $\begin{array}{ll}x^{\prime}, y^{\prime} & \text { Cartesian coordinates }(\mathrm{m}) \\
y & \text { Dimensionles s coordinate }(\mathrm{m})\end{array}$ \\
\hline$T_{w}^{\prime}$ & Temperature of the plate $(\mathrm{K})$ & Greek symbols: \\
\hline$G r$ & Grashof number for heat transfer & $\boldsymbol{v} \quad$ Kine matic viscosity $\left(\mathrm{m}^{2} \cdot \mathrm{s}^{-1}\right)$ \\
\hline$g$ & Acceleration due to gravity $\left(\mathrm{m} . \mathrm{s}^{-2}\right)$ & \multirow[b]{2}{*}{$\left(\mathrm{W} \cdot \mathrm{m}^{-1} \cdot \mathrm{K}^{-1}\right)$} \\
\hline$t_{o}$ & Characteristic time (s) & \\
\hline $\begin{array}{l}D_{B} \\
K r\end{array}$ & $\begin{array}{l}\text { Chemical molecular diffusivity }\left(\mathrm{m}^{-} \mathrm{s}\right) \\
\text { Chemical reaction parameter }\end{array}$ & Thermal conductivity of the nanofluid \\
\hline$C_{w}^{\prime}$ & Concentration in the fluid at the plate & $\left(\mathrm{W} \cdot \mathrm{m}^{-1} \cdot \mathrm{K}^{-1}\right)$ \\
\hline & $\left(\mathrm{kg} \cdot \mathrm{m}^{-3}\right)$ & Kinematic viscosity of the base fluid $\left(\mathrm{m}^{2} . \mathrm{s}^{-1}\right)$ \\
\hline
\end{tabular}




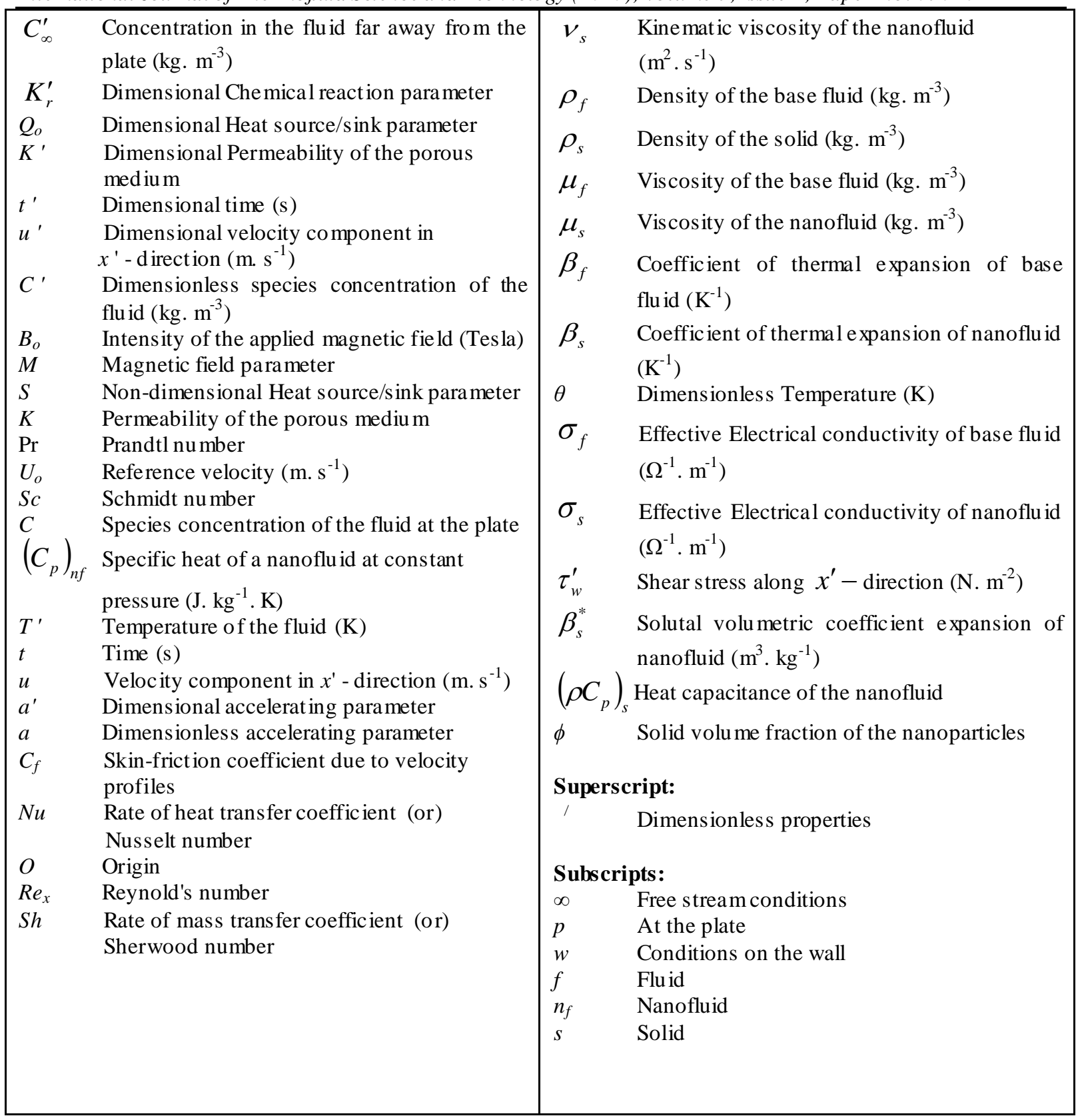

\section{References}

[1] Wenhua Yu, David M. France, Jules L. Routbort \& Stephen U. S. Choi, Review and comaprision of nanofluid thermal conductivity and heat transfer enhancements, Heat Transfer Eng. 29(2008), 432-460.

[2] Puneet Rana,R Bhargava,O.A. Beg, Numerical solution for mixed convection boundary layer flow of a nanofluid along an inclined plate embedded in a porous medium, Computers and Mathematics with Applications 64 (2012), 2816-2832.

[3] W. A. Khan, A. Aziz, Natural convection flow of a nanofluid over a vertical plate with uniform surface heat flux, Int. J. Therm. Sci., 50 (7) (2011),1207-1214.

[4] N. Putra, W. Roetzel, S.K. Das, Natural convection of nano-fluids, Int. J. Heat Mass Transfer 39 (2003), 775-784. 
[5] Kuznetsov, A., Nield, D., Natural convective boundary-layer flow of a nanofluid past a vertical plate, Int. J. Therm. Sci., 49 (2010), 243-247.

[6] R. S. R. Gorla, A. J. Chamkha, Natural convective boundary layer flow over a horizontal plate embedded in a porous medium saturated with a nanofluid, J. Mod. Phys., 2 (2011), 62-71.

[7] M. R. Krishnamurthy, B.C. Prasannakumar, B.J. Gireesha and Rama S.R. Gorla, Effect of Viscous dissipation on hydromagnetic fluid flow and heat transfer of nanofluid over an exponentially stretched sheet with fluid particle suspension, Cogent Mathematics (2015),2:1050973

[8] W. A. Khan, I. Pop, Boundary- layer flow of a nanofluid past a stretching sheet, Int. J. Heat Mass Transf., 53 (2010), 2477-2483.

[9] Job mackolil, Basavarajappa Mahenthesh, Exact and statistical computations of radiated flow of nano and cassion flow under heat and mass flux conditions, Journal of computational and design Engineering 6 (2019),593-605.

[10] J. Raza, A. M. Rohni, Z. Omar, Md. Awais, Heat and mass transfer analysis of MHD nanofluid flow in a rotating channel with slip effects, Journal of Molecular Liquids, 219(2016), 703-708.

[11] A. Aziz, W. A. Khan, Natural convective boundary layer flow of a nanofluid past a convectively heated vertical plate, Int. J. Therm. Sci., 52 (2012), 83-90.

[12] B. Ganga, S. Mohamed Yusuff Ansari, N. Vishnu Ganesh, A.K. Abdul Hakeem, MHD flow of Boungiorno model nanofluid over a vertical plate with internal heat generation/absorption, Propulsion and Power Research, 5(2016), 211-222.

[13] F. Ali, M. Gohar, I. Khan, MHD flow of water-based Brinkman type nanofluid over a vertical plate embedded in a porous medium with variable surface velocity, temperature and concentration, Journal of Molecular Liquids, 223(2016), 412-419.

[14] M. S. Kandelousi, "KKL correlation for simulation of nanofluid flow and heat transfer in a permeable channel," Physics Letters A, 378(2014), 3331-3339.

[15] R. K. Tiwari and M. K. Das, "Heat transfer augmentation in a two-sided lid-driven differentially heated square cavity utilizing nanofluids, "International Journal of Heat and Mass Transfer, 50(2007), 2002-2018.

[16] M. Sheikholeslami, H. R. Ashorynejad, G. Domairry and I. Hashim, Flow and heat transfer of cu-water nanofluid between a stretching sheet and a porous surface in a rotating system, Journal of Applied Mathematics, 2012- 421320.

[17] M K Nayak, A K Abdul Hakeem, B Ganga, Influence of non-uniform heat source/sink and variable viscosity on mixed convection flow of third grade nanofluid over an inclined stretched Riga plate, International Journal of Thermofluid Science and Technology (2019),6(4):19060401

[18] A.K. Patra, M.K. Nayak, A. Misra, Viscosity of nanofluids-A Review, , International Journal of Thermofluid Science and Technology (2020), 7(2):070202.

[19] S. Mishra, M.K. Nayak, A. Misra, Thermal conductivity of nanofluids-A comprehensive review, , International Journal of Thermofluid Science and Technology (2020), 7(3):070301.

[20] M. K. Nayak , HHR impact on 3D radiative stretched flow of $\mathrm{Cu}-\mathrm{H} 2 \mathrm{O}$ nanofluid influenced by variable magnetic field and convective boundary condition. International Journal of Thermofluid Science and Technology (2019), 6(2):19060202 
[21] Kalidad Das, Amith Sarkar, Prabir Kumar kundu, Cu-water nanofluid flow induced by a vertical stretching sheet in presence of a magnetic field with convective heat transfer, Propulsion and Power Research, 2017;6(3):206-213.

[22] P. K. Pattnaik and T. Biswal, Analytical Solution of MHD Free Convective Flow through Porous Media with Time Dependent Temperature and Concentration, Walailak J. Sci. \& Tech., 12 (9) (2015),749-762.

[23] R. L. Hamilton and O. K. Crosser, Thermal conductivity of heterogeneous two component systems, Ind. Eng. Chem. Fundamen., 1 (1962),187-191.

[24] K. J. Bathe, Finite Element Procedures (Prentice-Hall, New Jersy), 1996.

[25] J. N. Reddy, An Introduction to the Finite Element Method (McGraw-Hill, New York), 1985. 